\title{
Commentary: Streamlining endovascular interventions on extracorporeal life support
}

\author{
Mark Helmers, MD, and Pavan Atluri, MD
}

\author{
From the Division of Cardiovascular Surgery, Department of Surgery, University of Pennsylvania, Philadelphia, \\ $\mathrm{Pa}$. \\ Disclosures: Authors have nothing to disclose with regard to commercial support. \\ Received for publication April 1, 2019; accepted for publication April 1, 2019; available ahead of print May 15, \\ 2019 . \\ Address for reprints: Pavan Atluri, MD, Division of Cardiovascular Surgery, Hospital of the University of Penn- \\ sylvania, 3400 Spruce St, 6 Silverstein Pavilion, Philadelphia, PA (E-mail: Pavan.Atluri@uphs.upenn.edu). \\ J Thorac Cardiovasc Surg 2019;158:1368-9 \\ $0022-5223 / \$ 36.00$ \\ Copyright (c) 2019 by The American Association for Thoracic Surgery \\ https://doi.org/10.1016/j.jtcvs.2019.04.014
}

Since Hill and colleagues' initial description of successful implementation of extracorporeal membrane oxygenation (ECMO) in a 24-year-old man who developed respiratory failure after repair of a traumatic thoracic aortic transection in $1972,{ }^{1}$ the use of ECMO has exploded. In the United States alone, ECMO cases increased $433 \%$ from 2006 to 2011, in clinical scenarios ranging from acute respiratory distress syndrome, to cardiogenic shock and ECMOassisted cardiopulmonary resuscitation, to bridging patients to thoracic organ transplantation or durable mechanical circulatory support platforms. ${ }^{2-4}$ These critically ill patients often require endovascular interventions, such as percutaneous coronary intervention or catheter-directed thrombolytics. However, vascular access might be limited secondary to vasoconstriction, indwelling lines, or other mechanical circulatory support devices. Because of the need for anticoagulation, bleeding complications plague greater than $40 \%$ of ECMO patients..$^{5}$ Additional vascular access sites might further increase this bleeding risk.

In this issue of The Journal of Thoracic and Cardiovascular Surgery, Pasrija and colleagues describe a novel adaptor that splices into the outflow limb of ECMO circuits to enable endovascular access without need for additional arterial puncture or venipuncture. ${ }^{6}$ Their design incorporates a hemostatic valve, which allows passage of wires and catheters up to 8 French (Fr) in size, allows for deairing of the circuit, and accommodates an obturator, which prevents flow stagnation.

The authors performed computation flow analysis with their adaptor and a 19-Fr arterial cannula, which demonstrated laminar flow without stagnation. In vitro analysis using a glycerol solution was used to test varying sizes (15-23 Fr) of arterial cannulae for flow and pressure parameters without and with introduction of a 5-Fr catheter through the novel adaptor. Although introduction of the 5-Fr catheter resulted in a $24 \%$ decrement in circuit flow and an associated $16 \%$ increase in arterial pressure in a $15-\mathrm{Fr}$ arterial cannula, only $5 \%$ decrement in flow with a $10 \%$ pressure

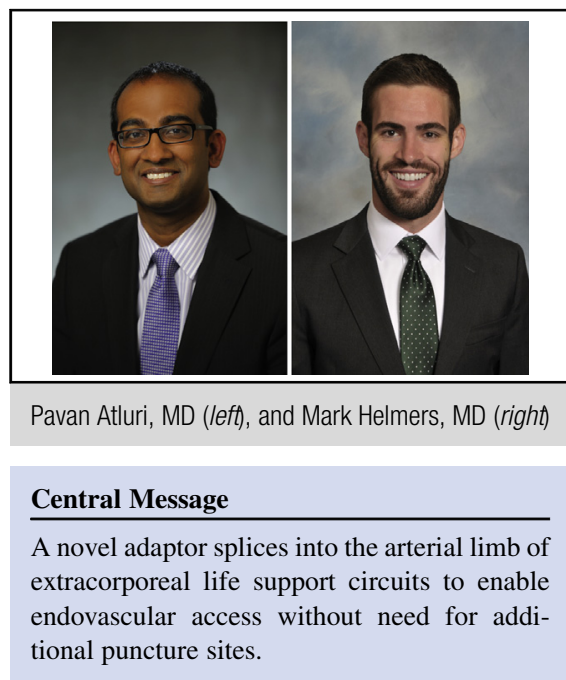

See Article page 1359. increase was seen with a 23-Fr cannula. The authors suggest these can be easily overcome with modest increases in pump speed. They demonstrated the feasibility of their system by performing left ventriculography, aortography, coronary angiography, and placement of a guide wire into the left anterior descending and circumflex arteries via femoral and carotid arterial access in porcine models.

There are important considerations that deserve attention. First, although the meticulous device design has minimized flow stagnation, there is potential for ring thrombus formation similar to tubing connectors. Although no thrombus was observed during the short in vivo run, further experimentation and careful monitoring is warranted. Second, as the authors indicated, use of this device on the venous inflow portion of a circuit should not be performed because the negative pressure generated from centrifugal flow pumps can result in catastrophic air entrainment. Last, hemolysis might result when larger catheters are inserted through smaller cannulas.

Nevertheless, Pasrija and colleagues should be commended for their novel design, ${ }^{6}$ which will invariably simplify intervention in a growing, critically ill patient population. Emerging data in the field of ECMO-assisted cardiopulmonary resuscitation has shown that percutaneous coronary intervention is associated with a mortality benefit in this population. ${ }^{7}$ Furthermore, as the ECMO field continues to grow, more attention in being paid to nuances, 
including the importance of venting of the left ventricle. ${ }^{8,9}$ This device could permit minimally invasive transaortic, catheter-based venting. ${ }^{10}$

\section{References}

1. Hill JD, O’Brien TG, Murray JJ, Dontigny L, Bramson ML, Osborn JJ, et al. Prolonged extracorporeal oxygenation for acute post-traumatic respiratory failure (shock-lung syndrome). N Engl J Med. 1972;286:629-34.

2. Sauer CM, Yuh DD, Bonde P. Extracorporeal membrane oxygenation use has increased by $433 \%$ in adults in the United States from 2006 to 2011. ASAIO J. 2015;61:31-6.

3. Fux T, Holm M, Corbascio M, Lund LH, van der Linden J. Venoarterial extracorporeal membrane oxygenation for postcardiotomy shock: risk factors for mortality. J Thorac Cardiovasc Surg. 2018;156:1894-902.e3.

4. Benazzo A, Schwarz S, Frommlet F, Schweiger T, Jaksch P, Schellongowski P, et al. Twenty-year experience with extracorporeal life support as bridge to lung transplantation. J Thorac Cardiovasc Surg. 2019 [In press].

5. Cheng R, Hachamovitch R, Kittleson M, Patel J, Arabia F, Moriguchi J, et al. Complications of extracorporeal membrane oxygenation for treatment of cardiogenic shock and cardiac arrest: a meta-analysis of 1,866 adult patients Ann Thorac Surg. 2014;97:610-6.

6. Pasrija C, Bittle GJ, Zhang J, Morales D, Tran D, Deatrick KB, et al. A novel adaptor system enables endovascular access through extracorporeal life support circuits. J Thorac Cardiovasc Surg. 2019;158:1359-66.

7. Sung K, Yang JH, Choi SH, Song YB, Yang JH, Hahn JY, et al. Two-year survival and neurological outcome of in-hospital cardiac arrest patients rescued by extracorporeal cardiopulmonary resuscitation. Int $J$ Cardiol. 2013;168:3424-30.

8. Cevasco M, Takayama H, Ando M, Garan AR, Naka Y, Takeda K. Left ventricular distension and venting strategies for patients on venoarterial extracorporeal membrane oxygenation. J Thorac Dis. 2019 [In press].

9. Distelmaier K, Wiedemann D, Binder C, Haberl T, Zimpfer D, Heinz G, et al. Duration of extracorporeal membrane oxygenation support and survival in cardiovascular surgery patients. J Thorac Cardiovasc Surg. 2018;155 2471-6.

10. Hong TH, Byun JH, Yoo BH, Hwang SW, Kim HY, Park JH. Successful left-heart decompression during extracorporeal membrane oxygenation in an adult patient by percutaneous transaortic catheter venting. Korean J Thorac Cardiovasc Surg. 2015;48:210-3. 